and "check and do" list if there is not already one in use. Our experience showed that significant reduction in CLABSI rate can be achieved in a relatively small NNU, over a relatively short time scale with similar effectiveness to that shown in larger NNUs and with close to zero additional costs.

\section{G538(P) REDUCING PRESCRIBING ERRORS BY INTRODUCING A PAEDIATRIC PRESCRIBING WEB-BASED LEARNING MODULE IN A TERTIARY CHILDREN'S HOSPITAL}

$1,{ }^{2} \mathrm{~V}$ Monnelly, ${ }^{2} \mathrm{~S}$ Kiff, ${ }^{2} \mathrm{R}$ Ardill. ' Neonatal Unit, Royal Infirmary of Edinburgh, Edinburgh, UK; ${ }^{2}$ General Paediatrics, Royal Hospital for Sick Children, Edinburgh, UK

\subsection{6/archdischild-2015-308599.489}

Background Medication errors are a significant problem in theUK, and have the potential to cause harm. Children are particularly vulnerable. Errors occur at different decision-making points in the medicines pathway, and prescribing errors constitute a significant but potentially modifiable step in this process, which we targeted.

Our hospital regularly audits prescribing against our local prescribing policy (the gold standard), which is based upon GMC guidance for safe prescribing. The rate of prescription error was $67 \%$ in 2010. We defined 'prescription error' as any deviation from the gold standard.

From August 2010, our mandatory junior doctor induction was modified to include a $2 \mathrm{~h}$ lecture on paediatric prescribing (delivered by pharmacist and doctor) and a paediatric prescribing test. This combination was shown to reduce prescribing errors from $67 \%$ to $52 \%$, and reduce incorrect or absent allergy information on prescription charts from $36 \%$ to $15 \%$ in 2010 and 2012 respectively.

The problem Although effective in improving prescribing, the lecture had a large volume of information to deliver in a limited time. Administering and marking the test was time consuming and providing individual feedback was logistically challenging.

A potential solution Web-based learning modules (WBLM) are accessible, low cost and can facilitate simultaneous education and assessment.

Aim To design and pilot a WBLM to replicate the previous content of prescribing induction. The long-term aim is to continue to improve paediatric prescribing within our hospital.
Primary objective Assess feasibility of introducing a WBLM as part of paediatric induction.

Secondary objective Assess the effect of the WBLM on prescribing practice.

The intervention The WBLM was designed by paediatricians (the authors) working with a Learnpro designer. It can be accessed remotely and is PC or tablet compatible.

There are 3 main sections. The first is an introduction to paediatric prescribing, revising pharmacokinetics and local policy for safe prescribing. The second section uses clinical cases to illustrate prescribing challenges and focuses on areas where errors frequently occur. Adverse drug reactions, antibiotic monitoring, medication error reporting and discharge prescriptions are covered. The final section focuses on intravenous fluids.

All sections contain links to relevant local clinical guidelines. Summative assessment questions are incorporated into the module, requiring trainees to obtain the correct answer before progressing.

Implementation The WBLM was designed in early 2014. Usability was tested in a small group prior to a pilot in August 2014 for junior medical and surgical doctors attending hospital induction.

Measurement of Improvement The Plan-Do-Study-Act cycle (PDSA) provides a structure for iterative testing of changes to improve quality. We are currently in the third cycle following the August 2014 pilot. All post-completion evaluations were positive. Minor technical issues reported have been addressed. The small scale testing has allowed adaptation according to feedback received.

All inpatient prescription charts, excluding PICU who use electronic prescribing, were audited in November 2014 (Figure 1).

There were 78 prescription charts containing 904 individual prescriptions. $85 \%$ of 'regular' prescriptions were prescribed correctly. Although only $68 \%$ of total prescriptions were correct, a significant proportion of errors were in 'once only' and 'PRN' sections, commonly completed in $\mathrm{A}+\mathrm{E}$ by doctors, who had not undertaken the WBLM. From 2015 the WBLM will be mandatory for all trainees rotating through the hospital, including $\mathrm{A}+\mathrm{E}$, anaesthesia and PICU.

Conclusion It is feasible to implement a WBLM as part of paediatric prescribing induction. Extending implementation to

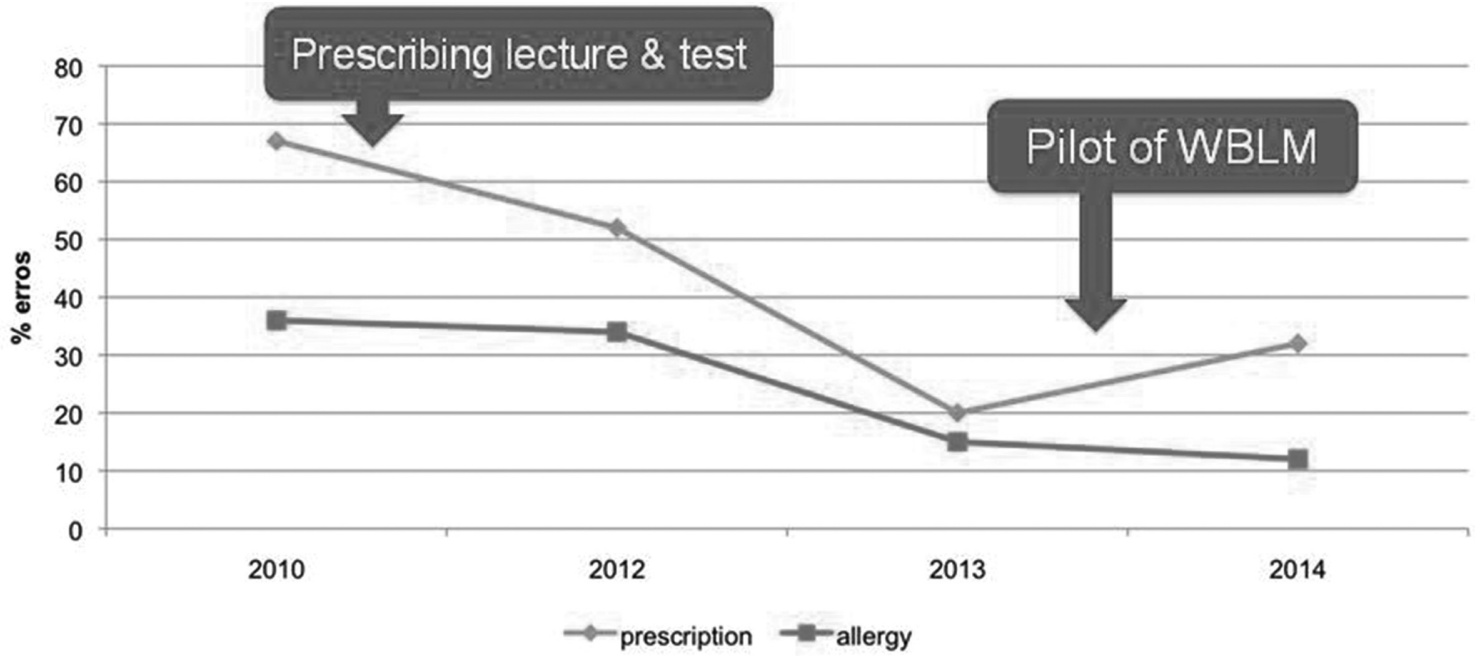

Abstract G538(P) Figure 1 Improving trends in prescription error and allergy documentation over time 
all hospital departments has the potential to be effective in improving paediatric prescribing practice. The WBLM must work in conjunction with other measures to address human and system factors in prescribing errors. These effects should be studied with ongoing PDSA cycles.

\section{G539(P) TRAINEE-LED QUALITY IMPROVEMENT IN ENHANCING SERVICE IN COMMUNITY PAEDIATRICS}

EC Ferguson, CL Enevoldson, C Harrison, S Hashmi, E Nagmeldin, S Suri. Department of Paediatrics, Rotherham NHS Foundation Trust, Rotherham, UK

\subsection{6/archdischild-2015-308599.490}

Context We planned to address two key areas of service provision in our secondary level hospital.

Firstly, in keeping with many Community Paediatric Departments, our hospital has experienced an increase in the number and complexity of referrals. As a result many families had long waits for consultations.

Secondly in our trust safeguarding work had historically represented a large proportion of the acute workload.

Problem and Assessment 1. Child Development Centre (CDC) appointments

Long waiting lists for medical appointments were negatively affecting patient care. The department was not compliant with the NICE guidance on the timeframe for assessment of children with possible social communication disorders. Patient satisfaction surveys had also identified this as an area of concern for families.

\section{Child Protection Assessments}

Vulnerable children competed with acute admissions for assessments and were often seen out of hours, when Consultant medical staff were not on site. Social Care had highlighted concerns about long and unpredictable waits for assessments.

Intervention Firstly, trainees in consultation with their consultant supervisors developed a flexible clinical arrangement to target service provision and meet community competencies in the CDC setting. Trainees were expected to complete six CDC clinics during their six month rotation, which were tailored to the trainee's acute rota.

Secondly, a daily child protection medical clinic was implemented and delivered by trainees under consultant supervision. Monthly safeguarding peer review meetings were initiated in line with Royal College of Paediatrics and Child Health guidance to provide support and facilitate training in this area.

Strategy for change To instigate and review the response to change, the Plan-Do-Study-Act (PDSA) cycle was used. An initial multi-disciplinary team (MDT) meeting was used to formalise plans and disseminate information, with three monthly MDT feedback meetings planned.

Measurement of improvement During the initial six month period, review of waiting lists has shown that trainee delivered CDC clinics have resulted in a reduction of waiting lists by 56 patients (the equivalent of 0.4 PA Consultant time). Urgent new patient appointments have fallen from 12 to 0 , although routine new patient appointments have increased from 45 to 68 . Feedback from the MDT is largely positive.

Review of child protection referrals has shown that following the instigation of child protection medical clinics the number of assessments occurring out of hours has fallen to 19\% compared to $44 \%$ previously. $81 \%$ of children are now seen at a prearranged time, without delay, compared to approximately $23 \%$ previously. Trainees report feeling more supported in carrying out safeguarding work.

A repeat CDC patient satisfaction survey and a planned audit of the child protection clinics will provide further feedback on the success of these interventions, which will lead to further action (PDSA).

Effects of changes Developing a flexible approach to encompass trainees' acute commitments, has required meticulous planning. Room availability was an unexpected factor needing close consideration in the plans. Involvement of trainees has required support and supervision both from Consultants, and the wider MDT, thus requiring some changes to their previous practice.

Lessons learnt Managing a change in service provision requires consideration of many different priorities. When trainees take a key role, it ensures the changes work at their level.

Message for others Working within the European Working Time Directive and today's financial constraints, a flexible approach to service provision is necessary. This initiative has highlighted that direct trainee involvement in the design of service delivery can result in significant changes that benefit patients, trusts and trainees alike. Moreover this venture has enhanced community training, as well as providing trainees with valuable experience in management of change.

\section{G540(P) AN IMPROVEMENT PROJECT TO REDUCE CENTRAL LINE ASSOCIATED BLOOD STREAM INFECTION (CLABSI) IN NEWBORN INFANTS}

${ }^{1} S$ Mohamed Cassim, ${ }^{2} \mathrm{C}$ Skiffington, ${ }^{3} \mathrm{C}$ Lucas, ${ }^{1,2} \mathrm{D}$ Anand. ${ }^{1}$ Neonatology, The Royal Hospital for Sick Children Yorkhill, Glasgow, UK; ${ }^{2}$ Medicine, University of Glasgow, Glasgow, UK; ${ }^{3}$ Microbiology, The Royal Hospital for Sick Children Yorkhill, Glasgow, UK

\subsection{6/archdischild-2015-308599.491}

Context The work is being done in a level 3 neonatal unit providing intensive care for babies with surgical, cardiac, ENT and other complex problems requiring both neonatal and paediatric specialist input. All infants admitted between December 2010 and June 2014 with central lines (CL) and those that were sited during the admission period were included.

Problem There were concerns among staff regarding high rate of CLABSI and lack of standardised management of CL. This was resulting in increased morbidity and length of stay.

Assessment of problem and analysis of its causes The baseline CLABSI rate was quantified in the first 4 months by monitoring infection in all infants with CL. CLABSI was identified using pre-defined criteria. Champions were identified from all staff groups and a multidisciplinary team was formed. The practice of line insertion and maintenance; and published literature was reviewed to identify potential problems and areas for improvement. An improvement project charter was developed describing the aims and objectives, components of the care bundle and plan for measuring and monitoring of processes and outcomes.

Intervention The intervention was a care bundle comprising of 1) line trolley with standardised equipment 2) hand hygiene 3) standardised checklist of techniques for aseptic insertion and maintenance of CL 4) daily review of need for CL.

Study design The study was designed using improvement methodology. A base line rate of CLABSI per 1000 central line days (CLD) was monitored monthly during the first four months, followed by implementation of care bundle and monthly monitoring of CLABSI rates for rest of the study period.

Strategy for change Through teaching sessions and meetings, all staff were informed of the base line CLABSI rates and the 